\title{
Quantum Dynamical $R$-matrices and Quantum Frobenius Group
}

\author{
G.E.Arutyunov * \\ and \\ S.A.Frolov ${ }^{\dagger}$
}

\begin{abstract}
We propose an algebraic scheme for quantizing the rational Ruijsenaars-Schneider model in the $R$-matrix formalism. We introduce a special parameterization of the cotangent bundle over $G L(N, \mathbf{C})$. In new variables the standard symplectic structure is described by a classical (Frobenius) $r$-matrix and by a new dynamical $\bar{r}$-matrix. Quantizing both of them we find the quantum $L$-operator algebra and construct its particular representation corresponding to the rational Ruijsenaars-Schneider system. Using the dual parameterization of the cotangent bundle we also derive the algebra for the $L$-operator of the trigonometric Calogero-Moser system.
\end{abstract}

*Steklov Mathematical Institute, Vavilov 42, GSP-1, 117966, Moscow, Russia; arut@class.mi.ras.ru

†Steklov Mathematical Institute, Vavilov 42, GSP-1, 117966, Moscow, Russia; frolov@class.mi.ras.ru 


\section{Introduction}

As soon as the classical dynamical $r$-matrices first appeared [1] on the scene of integrable many body systems, the problem of their quantization became of a real interest. The main hope related to this problem is to find a new algebraic structure that ensures the integrability of the corresponding quantum models.

We recall [2] that having a finite-dimensional completely integrable system with the Lax representation $\frac{d L}{d t}=[M, L]$ one can always write the Poisson algebra of $L$-operators in the $r$-matrix form. However, in general, an $r$-matrix appears to be a nontrivial function of dynamical variables. At present the classical dynamical $r$-matrices are known for the rational, trigonometric [1, 3] and elliptic [4, 5] Calogero-Moser (CM) systems, as well as for their relativistic generalizations - rational, trigonometric [6, 7] and elliptic [8, 9] RuijsenaarsSchneider (RS) systems [10].

The problem of quantizing the dynamical $r$-matrices is rather nontrivial since, in general, such $r$-matrices do not satisfy a single closed equation of the Yang-Baxter type, from which they can be uniquely determined. Up to now there exists only one example of a quantum dynamical $R$-matrix related to the quantum spin CM system [11]. This $R$-matrix solves the Gervais-Neveu-Felder equation [19, 13] and has a nice interpretation in terms of quasi-Hopf algebras [14.

A natural way to understand the origin of dynamical $r$-matrices is to consider the Hamiltonian reduction procedure [3, 15]. Factorizing a free motion on an initial phase space by the action of some symmetry group we get nontrivial dynamics on the reduced space. The $r$-matrix appears in the Dirac bracket, which describes the phase structure of the reduced space. In our recent papers [16, 17] we obtained the elliptic RS model, being the most general one among the integrable systems of the CM and RS types, by using two different reduction schemes. In the first scheme, the affine Heisenberg double was used as the initial phase space and in the second one we considered the cotangent bundle over the centrally extended group of double loops.

The aim of this paper is to quantize the reduction scheme leading to the dynamical systems of the RS type. Although the most interesting is the spectral-dependent elliptic case [16, 17], to clarify the general approach in this paper we restrict ourselves to considering the simplest rational model.

Our construction is based on a special parameterization of the cotangent bundle over the group $G L(N, \mathbf{C})$. This parameterization is similar to the one considered in [18]. However, instead of Euler angles, we use another system of generators to parameterize the "momentum". These generators obey a quadratic Poisson algebra describing by two $r$-matrices $r$ and $\bar{r}$. The matrix $r$ solves the $N$-parametric classical Yang-Baxter equation and is related to the special Frobenius subgroup in $G L(N, \mathbf{C})$.

We define a special matrix function $L$ on $T^{*} G$ invariant with respect to the action of this Frobenius subgroup. We call this function the " $L$-operator" since the Poisson algebra of $L$ literally coincides with the one for the rational RS model [7]. Moreover, performing the Hamiltonian reduction leading to the RS model [19], one can recognize in $L$ the standard $L$-operator of the rational RS model.

Then we pass to the quantization. The quadratic Poisson algebra can be quantized by using the $R$-matrix approach [20, 21]. The compatibility of the corresponding quantum algebra implies the quantum Yang-Baxter equation for $R$ and some new equations involving $R$ and $\bar{R}$. We solve these equations and get an explicit form for $R$ and $\bar{R}$. Coming back to the 
original generators of $T^{*} G$ we recover the standard commutation relations of the quantum cotangent bundle.

We derive a new quadratic algebra which is satisfied by the "quantum" $L$-operator. The matrices $R$ and $\bar{R}$ come in this algebra in a nontrivial way. This explains the nature of the dynamical $r$-matrices (classical and quantum) as the composite objects constructed from the more elementary blocks $R$ and $\bar{R}$.

It follows from our construction that the quantum $L$-operator is factorized in the form $L=$ $W P$. Here $W$ satisfies the defining relations of the quantum Frobenius group, $W_{1} W_{2} R_{12}=$ $R_{12} W_{2} W_{1}$, where $R$, being the quantization of $r$, is the $N$-parametric solution to the quantum Yang-Baxter equation. The diagonal matrix $P$ plays the role of a generalized momentum. We find the simplest representation of the $L$-operator algebra and relate it with the rational RS model.

The trigonometric CM system is known to be dual to the rational RS model [10]. This duality is explained by the existence of the dual parameterization of $T^{*} G$. In this parameterization the $\mathrm{CM}$ model can be easily quantized and we get the commutation relations satisfied by the corresponding quantum $L$-operator.

\section{Frobenius algebra and dynamical $r$-matrices}

In this section we introduce a special parameterization of the cotangent bundle $T^{*} G$ over the matrix group $G=G L(N, \mathbf{C})$. As a manifold the space $T^{*} G$ is naturally isomorphic to $\mathcal{G}^{*} \times G$, where $\mathcal{G}^{*}$ is dual to the Lie algebra $\mathcal{G}=\operatorname{Mat}(N, \mathbf{C})$. The standard Poisson structure on $T^{*} G$ can be written in terms of variables $(A, g)$, where $A \in \mathcal{G}^{*} \approx \mathcal{G}$ and $g \in G$, as follows

$$
\begin{aligned}
\left\{A_{1}, A_{2}\right\} & =\frac{1}{2}\left[C, A_{1}-A_{2}\right] \\
\left\{A_{1}, g_{2}\right\} & =g_{2} C \\
\left\{g_{1}, g_{2}\right\} & =0
\end{aligned}
$$

Here we use the standard tensor notation and $C=\sum_{i, j} E_{i j} \otimes E_{j i}$ is the permutation operator.

Any matrix $A$ belonging to an orbit of maximal dimension in $\mathcal{G}^{*}$ admits the factorization:

$$
A=T Q T^{-1}
$$

where $Q$ is a diagonal matrix with entries $q_{i}, q_{i} \neq q_{j}$. We also fix the order of $q_{i}$ : $q_{1}<$ $q_{2} \ldots<q_{n}$ by using the action of the Weyl group. It is obvious that the matrix $T$ in eq.(2.4) is not uniquely defined. Indeed, one can multiply $T$ by an arbitrary diagonal matrix from the right. We remove this ambiguity by imposing the following condition:

$$
T e=e,
$$

where $e$ is a vector with all $e_{i}=1$. The choice of (2.5) is motivated by the study of the reduction procedure leading to the Calogero-type integrable systems [22]. Let us note that the condition (2.5) defines a Lie subgroup $F \subset G$. The corresponding Lie algebra $\mathcal{F}$ has the natural basis $F_{i j}=E_{i i}-E_{i j}$, where $E_{i j}$ are the standard matrix unities. The commutation relations of $F_{i j}$ are

$$
\left[F_{i j}, F_{k l}\right]=\delta_{i k}\left(F_{i l}-F_{i j}\right)+\delta_{i l}\left(F_{k j}-F_{k l}\right)+\delta_{j k}\left(F_{i j}-F_{i l}\right)
$$


It is worthwhile to mention that $\mathcal{F}$ is not only the Lie algebra but also an associative algebra with respect to the usual matrix multiplication:

$$
F_{i j} F_{k l}=\delta_{i k} F_{i l}+\delta_{j k}\left(F_{i k}-F_{i l}\right) .
$$

Let us now rewrite the Poisson structure (2.1) in terms of the variables $T$ and $Q$. It is obvious that the coordinates $q_{i}$ commute with $A, T$ and $Q$ since they belong to the center of the Poisson algebra (2.1). Thus, it is enough to calculate the Poisson bracket $\{T, T\}$. We have

$$
\left\{T_{i j}, T_{k l}\right\}=\sum_{m n, p s} \frac{\delta T_{i j}}{\delta A_{m n}} \frac{\delta T_{k l}}{\delta A_{p s}}\left\{A_{m n}, A_{p s}\right\} .
$$

To find $\frac{\delta T_{i j}}{\delta A_{m n}}$ we perform the variation of (2.4):

$$
T^{-1} \delta T Q-Q T^{-1} \delta T+\delta Q=T^{-1} \delta A T
$$

This equation can be easily solved, and we obtain the derivatives

$$
\frac{\delta T_{i j}}{\delta A_{m n}}=\sum_{a \neq j} \frac{1}{q_{j a}}\left(T_{i a} T_{n j} T_{a m}^{-1}+T_{i j} T_{n a} T_{j m}^{-1}\right)
$$

and

$$
\frac{\delta q_{i}}{\delta A_{m n}}=T_{n i} T_{i m}^{-1}
$$

where $q_{i j} \equiv q_{i}-q_{j}$.

By using (2.6) we get

$$
\left\{T_{1}, T_{2}\right\}=T_{1} T_{2} r_{12}(q)
$$

where the $r$-matrix

$$
r_{12}(q)=\sum_{i \neq j} \frac{1}{q_{i j}} F_{i j} \otimes F_{j i}
$$

appears. It is clear, that $r_{12}(q)$ should be a skew-symmetric solution of the classical YangBaxter equation (CYBE). The origin of this $r$-matrix can be easily understood if we note that $\mathcal{F}$ is a Frobenius Lie algebra, i.e. there is a nondegenerate 2-cocycle (coboundary) on $\mathcal{F}:$

$$
\omega(X, Y)=\operatorname{tr}(Q[X, Y]), \quad X, Y \in \mathcal{F} .
$$

According to [23], to any Frobenius Lie algebra one can associate a skew-symmetric solution of the CYBE by inverting the corresponding 2-cocycle. One can check that the cocycle (2.10) corresponds to $r_{12}(q) \in \mathcal{F} \wedge \mathcal{F}$. Coming back to (2.4) we see that any orbit of maximal dimension in $\mathcal{G}^{*}$ can be supplied with the structure of the Frobenius group. It is worthwhile to note that $\omega$ is the Kirillov symplectic form on the coadjoint orbit of the maximal dimension parameterized by $Q$.

Now, following [18], we introduce a special parameterization for the group element $g$. Namely, we take $A^{\prime}=g A g^{-1}$, which Poisson commutes with $A$ and possesses the Poisson bracket

$$
\left\{A_{1}^{\prime}, A_{2}^{\prime}\right\}=-\frac{1}{2}\left[C, A_{1}^{\prime}-A_{2}^{\prime}\right]
$$

Diagonalizing it with the help of the matrix $U, U e=e, A^{\prime}=U Q U^{-1}$, we find that

$$
g=U P T^{-1}
$$


where $P$ is some diagonal matrix. It is obvious that the Poisson bracket for $U$ is given by

$$
\left\{U_{1}, U_{2}\right\}=-U_{1} U_{2} r_{12}(q)
$$

and that $\{T, U\}=0$. To proceed with the calculation of the brackets $\{U, P\},\{T, P\},\{P, P\}$ and $\{P, Q\}$ we should use the derivative $\frac{\delta U_{i j}}{\delta A_{m n}^{\prime}}$ which is given by (2.6) with the replacement $T \rightarrow U, A \rightarrow A^{\prime}$. After simple calculations we get

$$
\begin{aligned}
\left\{P_{1}, P_{2}\right\} & =0, \\
\left\{Q_{1}, P_{2}\right\} & =P_{2} \sum_{i} E_{i i} \otimes E_{i i} .
\end{aligned}
$$

Introducing $p_{i}=\log P_{i}$ we conclude that $\left\{q_{i}, p_{j}\right\}=\delta_{i j}$. We also find the remaining Poisson brackets

$$
\begin{aligned}
& \left\{U_{1}, P_{2}\right\}=U_{1} P_{2} \bar{r}_{12}(q), \\
& \left\{T_{1}, P_{2}\right\}=T_{1} P_{2} \bar{r}_{12}(q),
\end{aligned}
$$

where we have introduce a new matrix

$$
\bar{r}_{12}(q)=\sum_{i \neq j} \frac{1}{q_{i j}} F_{i j} \otimes E_{j j} .
$$

The Jacobi identity leads to a set of equations on the matrices $r$ and $\bar{r}$. However, we postpone the discussion of these equations till the next section, where the quantization of $T^{*} G$ will be given in terms of variables $Q, T, P, U$.

Let us define the $L$-operator as the following function of phase variables, being invariant under the action of the Frobenius group:

$$
L=T^{-1} g T=T^{-1} U P .
$$

By using (2.8), (2.12) and (2.13-2.15) one can easily find the Poisson brackets containing $L$ :

$$
\begin{aligned}
\left\{Q_{1}, L_{2}\right\} & =L_{2} \sum_{i} E_{i i} \otimes E_{i i} \\
\left\{T_{1}, L_{2}\right\} & =T_{1} L_{2} \bar{r}_{12}(q)-T_{1} r_{12}(q) L_{2}, \\
\left\{L_{1}, L_{2}\right\} & =r_{12}(q) L_{1} L_{2}+L_{1} L_{2}\left(\bar{r}_{12}(q)-\bar{r}_{21}(q)-r_{12}(q)\right) \\
& +L_{1} \bar{r}_{21}(q) L_{2}-L_{2} \bar{r}_{12}(q) L_{1} .
\end{aligned}
$$

We see that the brackets for $L$ and $Q$ are the brackets for the $L$-operator and coordinates of the rational RS model found in [7]. It is obvious that $I_{n}=\operatorname{tr} L^{n}=\operatorname{tr} g^{n}$ form a set of mutually commuting functions.

Let us note that the $L$-operator (2.17) has the form $L=W P$, where $W=T^{-1} U$. Since both $T$ and $U$ are elements of the Frobenius group $F, W$ also belongs to $F$. Calculating the Poisson bracket for $W$ we see that it coincides with the Sklyanin bracket defining on $F$ the structure of a Poisson-Lie group:

$$
\left\{W_{1}, W_{2}\right\}=\left[r_{12}(q), W_{1} W_{2}\right] .
$$

The Poisson relations of $W$ and $P$ are

$$
\left\{W_{1}, P_{2}\right\}=-P_{2}\left[\bar{r}_{12}(q), W_{1}\right] .
$$


The well-known property of the Poisson algebra (2.21) is the existence of a family of mutually commuting functions $J_{n}=\operatorname{tr} W^{n}$. Moreover, it turns out that $J_{n}$ commute not only with themselves but also with $P$ and $Q$. In Section 3 we show that the same property holds in the quantum case.

Now we construct the simplest representation of the Poisson algebra (2.21), (2.22) and (2.13) and relate it with the rational RS model. In fact, this representation corresponds to the zero dimensional symplectic leaf of the bracket (2.21).

To this end we employ the Hamiltonian reduction procedure. We recall that $G$ acts on $T^{*} G$ by the transformations $A \rightarrow h A h^{-1}, g \rightarrow h g h^{-1}$ in a Hamiltonian way. The corresponding moment map $\mu$ has the form $\mu=g A g^{-1}-A$. Performing the Hamiltonian reduction we fix it's value to be

$$
g A g^{-1}-A=-\gamma(e \otimes e-1)
$$

where $\gamma$ is an arbitrary constant. In terms of $(T, L, Q)$ variables this equation acquires the form

$$
T\left(L Q L^{-1}-Q\right) T^{-1}=-\gamma(e \otimes e-1) .
$$

Since $T e=e$ and $L=W P$ the last equation can be written as

$$
W Q-Q W-\gamma W=-\gamma e \otimes(e U)
$$

Eq.(2.24) can be elementary solved and one gets

$$
W=\sum_{i, j} \frac{\gamma}{\gamma+q_{i j}} e_{i} b_{j} E_{i j},
$$

where $b=e U$. If we recall that $W$ should be an element of $F$, i.e. $W e=e$, then we find the coefficients $b_{j}$ :

$$
b_{j}=\frac{1}{\gamma} \frac{\prod_{a}\left(q_{a j}+\gamma\right)}{\prod_{a \neq j} q_{a j}}
$$

and thereby

$$
W_{i j}=\frac{\prod_{a \neq i}\left(q_{a j}+\gamma\right)}{\prod_{a \neq j} q_{a j}} .
$$

One can check that $W$ given by (2.27) has the desired Poisson brackets (2.21) and (2.22). We do not give an explicit proof of this statement since in section 3 we show that the same function $W$ realizes the representation for the corresponding quantum algebra.

The relation of our $L$-operator with the standard Ruijsenaars $L$-operator 10 is given by the following canonical transformation:

$$
q_{i} \rightarrow q_{i}, \quad P_{i} \rightarrow \prod_{a \neq i} \frac{\left(q_{a i}-\gamma\right)^{1 / 2}}{\left(q_{a i}+\gamma\right)^{1 / 2}} P_{i}
$$

\section{Quantum L-operator algebra}

In this section we shall quantize $T^{*} G$ in terms of variables $Q, T, P, U$ and obtain the permutation relations for the quantum $L$-operator. The algebra of functions on $T^{*} G$ can be 
easily quantized and one gets an associative algebra generated by $A$ and $g$ subject to the standard relations:

$$
\begin{aligned}
{\left[A_{1}, A_{2}\right] } & =\frac{1}{2} \hbar\left[C, A_{1}-A_{2}\right] \\
{\left[A_{1}, g_{2}\right] } & =\hbar g_{2} C \\
{\left[g_{1}, g_{2}\right] } & =0
\end{aligned}
$$

where $\hbar$ is a quantization parameter. The commutation relations for the generators $T, Q, U, P$ can be straightforwardly written by using the ideology of the Quantum Inverse Scattering Method [20, 21] (we present only nontrivial relations):

$$
\begin{aligned}
T_{1} T_{2}= & T_{2} T_{1} R_{12}(q), \quad U_{1} U_{2}=U_{2} U_{1} R_{12}^{-1}(q) \\
T_{1} P_{2}= & P_{2} T_{1} \bar{R}_{12}(q), \quad U_{1} P_{2}=P_{2} U_{1} \bar{R}_{12}(q) \\
& {\left[Q_{1}, P_{2}\right]=\hbar P_{2} \sum_{i} E_{i i} \otimes E_{i i} . }
\end{aligned}
$$

Here $R(q)$ and $\bar{R}(q)$ are quantum dynamical $R$-matrices having the following behavior near $\hbar=0$ :

$$
R(q)=1+\hbar r(q)+o(\hbar), \quad \bar{R}(q)=1+\hbar \bar{r}(q)+o(\hbar)
$$

It follows from the compatibility conditions that the $R$-matrices should satisfy the following set of equations

$$
\begin{aligned}
R_{12}(q) R_{21}(q) & =1 \\
R_{12}(q) R_{13}(q) R_{23}(q) & =R_{23}(q) R_{13}(q) R_{12}(q) \\
R_{12}(q) \bar{R}_{13}(q) \bar{R}_{23}(q) & =\bar{R}_{23}(q) \bar{R}_{13}(q) P_{3}^{-1} R_{12}(q) P_{3} \\
\bar{R}_{12}(q) P_{2}^{-1} \bar{R}_{13}(q) P_{2} & =\bar{R}_{13}(q) P_{3}^{-1} \bar{R}_{12}(q) P_{3}
\end{aligned}
$$

Let us demonstrate how one gets, for example, eq.(3.36). This equation follows from (3.32) and the following chain of relations:

$$
\begin{gathered}
T_{1} T_{2} P_{3}=T_{1} P_{3} T_{2} \bar{R}_{23}=P_{3} T_{1} \bar{R}_{13} T_{2} \bar{R}_{23}=P_{3} T_{2} T_{1} R_{12} \bar{R}_{13} \bar{R}_{23}=T_{2} P_{3} \bar{R}_{23}^{-1} T_{1} R_{12} \bar{R}_{13} \bar{R}= \\
T_{2} T_{1} P_{3} \bar{R}_{13}^{-1} \bar{R}_{23}^{-1} R_{12} \bar{R}_{13} \bar{R}=T_{1} T_{2} R_{12}^{-1} P_{3} \bar{R}_{13}^{-1} \bar{R}_{23}^{-1} R_{12} \bar{R}_{13} \bar{R} .
\end{gathered}
$$

The solution of the Yang-Baxter equation (3.35) can be easily found if one notes that $r_{12}^{2}=0$. It is known (see e.g. [24] Prop.6.4.13) that if $r \in \operatorname{Mat}(N, \mathbf{C}) \otimes \operatorname{Mat}(N, \mathbf{C})$ satisfies $r^{3}=0$ and solves the classical Yang-Baxter equation then $R=e^{r}$ is a solution of QYBE. Therefore, $R_{12}=e^{\hbar r_{12}}=1+\hbar r_{12}$ is a desired solution of the QYBE.

The solution of eq.(3.36) can be found if one supposes that $\bar{R}$ has the same matrix structure as $\bar{r}$ does:

$$
\bar{R}_{12}(q)=1+\hbar \sum_{i \neq j} \bar{r}_{i j}(\hbar, q) F_{i j} \otimes E_{j j}
$$

Then the following $\bar{R}$-matrix is a solution of eqs. (3.36) and (3.37):

$$
\bar{R}_{12}(q)=1+\sum_{i \neq j} \frac{\hbar}{q_{i j}-\hbar} F_{i j} \otimes E_{j j} .
$$


It is not difficult to verify that $\bar{R}_{12}^{-1}(q)=1-\sum_{i \neq j} \frac{\hbar}{q_{i j}} F_{i j} \otimes E_{j j}$.

Now we should show that the generators $A=T Q T^{-1}$ and $g=U P T^{-1}$ satisfy the commutation relations (3.28 3.30$)$. From the relations (3.31-3.33) we get

$$
\begin{aligned}
{\left[A_{1}, A_{2}\right] } & =T_{2} T_{1}\left(R_{12} Q_{1} R_{21} Q_{2}-Q_{2} R_{12} Q_{1} R_{21}\right) T_{1}^{-1} T_{2}^{-1} \\
{\left[A_{1}, g_{2}\right] } & =g_{2} T_{2} T_{1}\left(R_{12}\left(Q_{1}+\hbar \sum_{i} E_{i i} \otimes E_{i i}\right) \bar{R}_{12}^{-1} R_{12}-R_{12} Q_{1}\right) T_{2}^{-1} T_{1}^{-1} \\
{\left[g_{1}, g_{2}\right] } & =U_{2} U_{1}\left(R_{12}^{-1} P_{1} \bar{R}_{21}^{-1} P_{2} \bar{R}_{12}^{-1} R_{12}-P_{2} \bar{R}_{12}^{-1} P_{1} \bar{R}_{21}^{-1}\right) T_{2}^{-1} T_{1}^{-1}
\end{aligned}
$$

By using the following identities that can be checked by direct computation

$$
\begin{aligned}
R_{12} Q_{1} R_{21} Q_{2}-Q_{2} R_{12} Q_{1} R_{21} & =\hbar\left(C Q_{1} R_{21}-R_{12} Q_{1} C\right) \\
R_{12}\left(Q_{1}+\hbar \sum_{i} E_{i i} \otimes E_{i i}\right) \bar{R}_{12}^{-1} R_{12}-R_{12} Q_{1} & =\hbar C \\
R_{12}^{-1} P_{1} \bar{R}_{21}^{-1} P_{2} \bar{R}_{12}^{-1} R_{12}-P_{2} \bar{R}_{12}^{-1} P_{1} \bar{R}_{21}^{-1} & =0
\end{aligned}
$$

one derives the desired commutation relations for $A$ and $g$. Let us finally present the permutation relations for $Q, T$ and $L$, which can be easily obtained by using (3.31-3.33) and (3.40):

$$
\begin{aligned}
{\left[Q_{1}, L_{2}\right] } & =\hbar L_{2} \sum_{i} E_{i i} \otimes E_{i i}, \\
T_{1} R_{12} L_{2} & =L_{2} T_{1} \bar{R}_{12} \\
L_{1} \bar{R}_{21}^{-1} L_{2} \bar{R}_{12}^{-1} R_{12} \bar{R}_{21} & =R_{12} L_{2} \bar{R}_{12}^{-1} L_{1}
\end{aligned}
$$

It is clear that just as in the classical case the quantities $I_{n}=\operatorname{tr} g^{n}$ form a set of mutually commuting operators. Let us show that $I_{n}$ can be expressed in terms of $L$ and $Q$ solely and thereby they can be interpreted as the quantum integrals of motion for the rational RS model. By using the definition of $L$ we rewrite $I_{n}$ as

$$
I_{n}=\operatorname{tr} g^{n}=\operatorname{tr} T L^{n} T^{-1}=\operatorname{tr}_{12} C_{12} T_{1} L_{1}^{n} T_{2}^{-1}=\operatorname{tr}_{12} C_{12}^{t_{2}} T_{1} L_{1}^{n} \stackrel{t}{T_{2}^{-1}}
$$

where $t_{2}$ denotes the matrix transposition in the second factor of the tensor product.

It follows from (3.42) that

$$
L_{1} \stackrel{t}{T_{2}^{-1}}=\stackrel{t}{T_{2}^{-1}} R_{12}^{t_{2}} L_{1} \bar{R}_{21}^{t_{2}}
$$

Applying this relation we derive

$$
I_{n}=\operatorname{tr}_{12} C_{12}^{t_{2}} T_{1} \stackrel{t}{-1}_{2}^{-1} R_{12}^{t_{2}} L_{1} \bar{R}_{21}^{t_{2}} \cdots R_{12}^{t_{2}} L_{1} \bar{R}_{21}^{t_{2}}
$$

Exchanging $T_{1}$ and $\stackrel{t}{T}_{2}^{-1}$ with the help of eq.(3.31) one gets

$$
I_{n}=\operatorname{tr}_{12} C_{12}^{t_{2}} \stackrel{t}{T_{2}^{-1}} T_{1} L_{1} \bar{R}_{21}^{t_{2}} \cdots R_{12}^{t_{2}} L_{1} \bar{R}_{21}^{t_{2}} .
$$

Since $C_{12}^{t_{2}} \stackrel{t}{T_{2}^{-1}} T_{1}=C_{12}^{t_{2}}$ and $\bar{R}_{21}^{t_{2}} C_{12}^{t_{2}}=C_{12}^{t_{2}}$ we finally arrive at

$$
I_{n}=\operatorname{tr}_{12} C_{12}^{t_{2}} L_{1} \bar{R}_{21}^{t_{2}} R_{12}^{t_{2}} L_{1} \bar{R}_{21}^{t_{2}} R_{12}^{t_{2}} L_{1} \cdots L_{1} \bar{R}_{21}^{t_{2}} R_{12}^{t_{2}} L_{1} .
$$


It is natural to regard this expression for $I_{n}$ as a "quantum trace" of the operator $L^{n}$.

Just as in the classical case the quantum $L$-operator has the form $L=W P$, where $W=T^{-1} U$ satisfies the defining relations of the quantum Frobenius group:

$$
R_{12} W_{2} W_{1}=W_{1} W_{2} R_{12}
$$

The algebra (3.41), (3.43) rewritten in terms of $Q, P$ and $W$ is given by (3.33), (3.44) and by the relation

$$
W_{1} P_{2} \bar{R}_{12}^{-1}=P_{2} \bar{R}_{12}^{-1} W_{1}
$$

This shows that the representation theory for $L$ essentially reduces to the one for the quantum Frobenius group.

It is known that the algebra (3.44) admits a family of mutually commuting operators given by [25]:

$$
J_{n}=\operatorname{tr}_{1 \ldots n}\left[\hat{R}_{12} \hat{R}_{23} \ldots \hat{R}_{n-1, n} W_{1} \ldots W_{n}\right],
$$

where $\hat{R}_{i j}=R_{i j} C_{i j}$. Now we demonstrate that $J_{n}$ commutes with $P$. For the sake of clarity we do it for $n=3$. It follows from (3.45) that

$$
J_{3} P_{4}=\hat{R}_{12} \hat{R}_{23} P_{4} \bar{R}_{14}^{-1} W_{1} \bar{R}_{14} \bar{R}_{24}^{-1} W_{2} \bar{R}_{24} \bar{R}_{34}^{-1} W_{3} \bar{R}_{34}
$$

Equation (3.36) written in terms of $\hat{R}$ acquires the form

$$
P_{3}^{-1} \hat{R}_{12}(q) P_{3}=\bar{R}_{23}^{-1} \bar{R}_{13}^{-1} \hat{R}_{12} \bar{R}_{13} \bar{R}_{23}
$$

By using this equation we can push $P_{4}$ on the left. Taking into account that $\bar{R}_{12}$ is diagonal in the second space we get

$$
J_{3} P_{4}=P_{4} \bar{R}_{14}^{-1} \bar{R}_{24}^{-1} \bar{R}_{34}^{-1} \hat{R}_{12} \hat{R}_{23} W_{1} W_{2} W_{3} \bar{R}_{34} \bar{R}_{24} \bar{R}_{14}
$$

Taking the trace in the first, second and third spaces one gets the desired property.

Now we give an example of the representation of the algebra (3.44) and (3.45). Namely, we prove that the $W$-operator given by eq.(2.27) realizes this algebra with $P_{j}=e^{-\hbar \frac{\partial}{\partial q_{j}}}$.

It is obvious that $\left[W_{1}, W_{2}\right]$ should be equal to zero since $W$ depends only on the coordinates $q_{i}$. Thus, the following relation has to be valid: $\left[r_{12}(q), W_{1} W_{2}\right]=0$. Substituting the explicit form of $r_{12}(q)$ we have

$$
\begin{aligned}
{\left[r_{12}(q), W_{1} W_{2}\right]_{k l m n} } & =W_{k l}\left(\frac{1}{q_{k m}} W_{m n}-\frac{1}{q_{k m}} W_{k n}-\frac{1}{q_{l n}} W_{m n}\right) \\
& -W_{m l}\left(\frac{1}{q_{k m}} W_{m n}-\frac{1}{q_{k m}} W_{k n}-\frac{1}{q_{l n}} W_{k n}\right) \\
& +\delta_{l n} \sum_{j \neq l} \frac{1}{q_{l j}}\left(W_{k l} W_{m j}-W_{k j} W_{m l}\right) .
\end{aligned}
$$

First we show that when $l \neq n$ the first line in (3.46) cancels the second one. Since $W_{i j}=\frac{\gamma}{\gamma+q_{i j}} b_{j}$ we get

$$
\begin{aligned}
& \frac{\gamma}{\gamma+q_{k l}}\left(\frac{1}{q_{k m}} \frac{\gamma}{\gamma+q_{m n}}-\frac{1}{q_{k m}} \frac{\gamma}{\gamma+q_{k n}}-\frac{1}{q_{l n}} \frac{\gamma}{\gamma+q_{m n}}\right)- \\
& \frac{\gamma}{\gamma+q_{m l}}\left(\frac{1}{q_{k m}} \frac{\gamma}{\gamma+q_{m n}}-\frac{1}{q_{k m}} \frac{\gamma}{\gamma+q_{k n}}-\frac{1}{q_{l n}} \frac{\gamma}{\gamma+q_{k n}}\right)=0
\end{aligned}
$$


In the case $l=n$ the r.h.s. of (3.46) reduces to

$$
\begin{aligned}
& -\frac{1}{q_{k m}}\left(W_{k l}-W_{m l}\right)^{2}+\sum_{j \neq l} \frac{1}{q_{l j}}\left(W_{k l} W_{m j}-W_{k j} W_{m l}\right)= \\
& -\frac{\gamma^{2} q_{k m}}{\left(\gamma+q_{k m}\right)^{2}\left(\gamma+q_{m l}\right)^{2}} b_{l}^{2}+\sum_{j \neq l} \frac{1}{q_{l j}}\left(\frac{\gamma}{\gamma+q_{k l}} \frac{\gamma}{\gamma+q_{m j}}-\frac{\gamma}{\gamma+q_{k j}} \frac{\gamma}{\gamma+q_{m l}}\right) b_{j} b_{l} \\
& =\frac{q_{m k}}{\gamma+q_{m l}} W_{k l} \sum_{j} \frac{W_{m j}}{\gamma+q_{k j}} .
\end{aligned}
$$

Thus, one has to show that the series $S=\sum_{j} \frac{W_{m j}}{\gamma+q_{k j}}$ vanishes. To this end we consider the following integral 円

$$
I=\frac{1}{2 \pi i} \oint \frac{d z}{q_{k}-z+\gamma} \frac{\prod_{a \neq m}\left(q_{a}-z+\gamma\right)}{\prod_{a}\left(q_{a}-z\right)},
$$

where the integration contour is taken around infinity. Since the integrand is nonsingular at $z \rightarrow \infty$, we get $I=0$. On the other hand, summing up the residues one finds $I=$ $\sum_{j} \frac{1}{\gamma+q_{k j}} \frac{\prod_{a \neq m}\left(q_{a j}+\gamma\right)}{\prod_{a \neq j} q_{a j}}=S$.

Now we turn to eq.(3.45). Explicitly it reads as

$$
\begin{aligned}
P_{j}^{-1}\left[W_{k l}, P_{j}\right] & =\left(\frac{\hbar}{q_{l j}-\hbar}-\frac{\hbar}{q_{k j}}-\frac{\hbar^{2}}{q_{k j}\left(q_{l j}-\hbar\right)}\right) W_{k l}+\left(\frac{\hbar^{2}}{q_{k j}\left(q_{l j}-\hbar\right)}+\frac{\hbar}{q_{k j}}\right) W_{j l} \\
& +\delta_{j l} \sum_{i \neq j}\left(\left(\frac{\hbar^{2}}{q_{k j}\left(q_{i j}-\hbar\right)}-\frac{\hbar}{q_{i j}-\hbar}\right) W_{k i}-\frac{\hbar^{2}}{q_{k j}\left(q_{i j}-\hbar\right)} W_{l i}\right) .
\end{aligned}
$$

For the sake of shortness in (3.47) we adopt a convention that if in some denominator $q_{i j}$ becomes zero, the corresponding fraction is also regarded as zero. Thus, eq.(3.47) is equivalent to the following system of equations

$$
\begin{aligned}
P_{j}^{-1}\left[W_{k l}, P_{j}\right] & =\frac{\hbar}{q_{k j}\left(q_{l j}-\hbar\right)}\left(q_{k l} W_{k l}-q_{j l} W_{j l}\right), \text { for } k \neq l \neq j ; \\
P_{j}^{-1}\left[W_{j l}, P_{j}\right] & =\frac{\hbar}{\left(q_{l j}-\hbar\right)} W_{j l}, \text { for } j \neq l ; \\
P_{k}^{-1}\left[W_{k k}, P_{k}\right] & =-\sum_{i \neq k} \frac{\hbar}{q_{i k}-\hbar} W_{k i} ; \\
P_{j}^{-1}\left[W_{k j}, P_{j}\right] & =\frac{\hbar}{q_{k j}}\left(W_{j j}-W_{k j}\right)+\frac{\hbar\left(\hbar-q_{k j}\right)}{q_{k j}} \sum_{i \neq j} \frac{1}{q_{i j}-\hbar} W_{k i} \\
& -\frac{\hbar^{2}}{q_{k j}} \sum_{i \neq j} \frac{1}{q_{i j}-\hbar} W_{j i}, \text { for } k \neq j .
\end{aligned}
$$

In the sequel we shall give an explicit proof only for the latter case since the other three cases are treated quite analogously. The 1.h.s. of (3.51) is

$$
P_{j}^{-1}\left[W_{k j}, P_{j}\right]=P_{j}^{-1} \frac{\prod_{a \neq k}\left(q_{a j}+\gamma\right)}{\prod_{a \neq j} q_{a j}} P_{j}-W_{k j}=\gamma \frac{\prod_{\substack{a \neq k \\ a \neq j}}\left(q_{a j}+\gamma-\hbar\right)}{\prod_{a \neq j}\left(q_{a j}-\hbar\right)}-W_{k j} .
$$

\footnotetext{
${ }^{1}$ We are gratefull to N.A.Slavnov for explaining us the technique of treating such series.
} 
As to the r.h.s., one needs to calculate the sum $\sum_{i \neq j} \frac{1}{q_{i j}-\hbar} W_{k i}$. For this purpose we evaluate the following integral with the integration contour around infinity:

$$
I=\frac{1}{2 \pi i} \oint \frac{d z}{z-q_{j}-\hbar} \frac{\prod_{a \neq k}\left(q_{a}-z+\gamma\right)}{\prod_{a}\left(q_{a}-z\right)},
$$

The regularity of the integrand at $z \rightarrow \infty$ gives $I=0$. On the other hand, summing up the residues one finds

$$
I=-\frac{1}{\hbar} \frac{\prod_{a \neq k}\left(q_{a j}+\gamma-\hbar\right)}{\prod_{a \neq j}\left(q_{a j}-\hbar\right)}-\sum_{i \neq j} \frac{1}{q_{i j}-\hbar} W_{k i}+\frac{1}{\hbar} W_{k j}
$$

from here one deduces the desired series:

$$
\sum_{i \neq j} \frac{1}{q_{i j}-\hbar} W_{k i}=-\frac{1}{\hbar} \frac{\prod_{a \neq k}\left(q_{a j}+\gamma-\hbar\right)}{\prod_{a \neq j}\left(q_{a j}-\hbar\right)}+\frac{1}{\hbar} W_{k j}
$$

and

$$
\sum_{i \neq j} \frac{1}{q_{i j}-\hbar} W_{j i}=-\frac{1}{\hbar} \frac{\prod_{a \neq j}\left(q_{a j}+\gamma-\hbar\right)}{\prod_{a \neq j}\left(q_{a j}-\hbar\right)}+\frac{1}{\hbar} W_{j j} .
$$

Now substituting these sums in the r.h.s. of (3.51) one proves (3.51).

It follows from our proof that the $L$-operator

$$
L=\sum_{i j} \frac{\prod_{a \neq i}\left(q_{a j}+\gamma\right)}{\prod_{a \neq j} q_{a j}} e^{-\hbar \frac{\partial}{\partial q_{j}}} E_{i j}
$$

realizes the representation of the algebra (3.43).

Let us briefly discuss the degeneration of the RS system to the rational CM model. To get the $\mathrm{CM}$ model one should rescale $\hbar \rightarrow \gamma \hbar$ and to consider the limit $\gamma \rightarrow 0, L \rightarrow 1+\gamma \mathcal{L}$. Then $\mathcal{L}$ is the $L$-operator of the CM model. From eqs.(3.41), (3.43) one derives the quantum algebra satisfied by the $L$-operator of the CM model:

$$
\begin{aligned}
& {\left[Q_{1}, \mathcal{L}_{2}\right]=\hbar \sum_{i} E_{i i} \otimes E_{i i}} \\
& {\left[\mathcal{L}_{1}, \mathcal{L}_{2}\right]=\hbar\left[r_{12}-\bar{r}_{12}, \mathcal{L}_{1}\right]-\hbar\left[r_{21}-\bar{r}_{21}, \mathcal{L}_{2}\right]+\hbar^{2}\left[r_{12}-\bar{r}_{12}, r_{21}-\bar{r}_{21}\right] .}
\end{aligned}
$$

The last formula can be written in the following elegant form

$$
\left[\mathcal{L}_{1}+\hbar\left(r_{21}-\bar{r}_{21}\right), \mathcal{L}_{2}+\hbar\left(r_{12}-\bar{r}_{12}\right)\right]=0 .
$$

\section{Quantum R-matrix for the trigonometric CM sys- tem}

In this section we describe the dual parameterization of $T^{*} G$, which is related to the trigonometric CM system. We start with diagonalizing the group element $g=V D V^{-1}$ and impose the constraint $V e=e$. The derivatives $\frac{\delta V_{i j}}{\delta g_{k l}}$ and $\frac{\delta D_{i}}{\delta g_{k l}}$ are obtained in the same manner as in Section 2. Calculating the Poisson brackets of $V, D$ and $A$ we get

$$
\begin{aligned}
\left\{V_{1}, A_{2}\right\} & =V_{1} V_{2} s_{12} V_{2}^{-1}, \\
\left\{D_{1}, A_{2}\right\} & =-D_{1} V_{2} \sum_{i} E_{i i} \otimes E_{i i} V_{2}^{-1},
\end{aligned}
$$


where

$$
s_{12}=-\sum_{i \neq j} \frac{D_{i}}{D_{i}-D_{j}} F_{i j} \otimes E_{j i} .
$$

The $\mathcal{L}$-operator corresponding to the trigonometric $\mathrm{CM}$ system will be given as the following function on the phase space:

$$
\mathcal{L}=V^{-1} A V
$$

Calculation of the Poisson algebra of $T^{*} G$ in terms of $\mathcal{L}, V, D$ results in

$$
\begin{aligned}
\left\{\mathcal{L}_{1}, \mathcal{L}_{2}\right\} & =\left[\tilde{r}_{12}, \mathcal{L}_{1}\right]-\left[\tilde{r}_{21}, \mathcal{L}_{2}\right] \\
\left\{V_{1}, \mathcal{L}_{2}\right\} & =V_{1} s_{12} \\
\left\{D_{1}, \mathcal{L}_{2}\right\} & =-D_{1} \sum_{i} E_{i i} \otimes E_{i i}
\end{aligned}
$$

where we have introduced the matrix

$$
\tilde{r}_{12}=-s_{12}+\frac{1}{2} C
$$

The matrix $\tilde{r}_{12}$ can be written in the following form

$$
\tilde{r}_{12}=-\frac{1}{2} \sum_{i \neq j} \operatorname{cth} \frac{q_{i j}}{2} E_{i j} \otimes E_{j i}+\frac{1}{2} \sum_{i \neq j} \frac{e^{\frac{q_{i j}}{2}}}{\sinh \frac{q_{i j}}{2}} E_{i i} \otimes E_{j i}+\frac{1}{2} \sum_{i} E_{i i} \otimes E_{i i},
$$

where $q_{i}=\log D_{i}$.

Now we clarify the connection of $\tilde{r}_{12}$ with the dynamical $r$-matrix first found in [四]. By conjugating $\mathcal{L}$-operator $(4.59)$ with the matrix $D^{\frac{1}{2}}: \tilde{\mathcal{L}}=D^{\frac{1}{2}} \mathcal{L} D^{-\frac{1}{2}}$ and calculating the Poisson bracket for $\tilde{\mathcal{L}}$ with the help of (4.60) and (4.62) we arrive at

$$
\left\{\tilde{\mathcal{L}}_{1}, \tilde{\mathcal{L}}_{2}\right\}=\left[\tilde{R}_{12}, \tilde{\mathcal{L}}_{1}\right]-\left[\tilde{R}_{21}, \tilde{\mathcal{L}}_{2}\right]
$$

where

$$
\begin{aligned}
\tilde{R}_{12} & =D_{1}^{\frac{1}{2}} D_{2}^{\frac{1}{2}} \tilde{r}_{12} D_{1}^{-\frac{1}{2}} D_{2}^{-\frac{1}{2}}-\frac{1}{2} \sum_{i} E_{i i} \otimes E_{i i} \\
& =-\frac{1}{2} \sum_{i \neq j} \operatorname{cth} \frac{q_{i j}}{2} E_{i j} \otimes E_{j i}+\frac{1}{2} \sum_{i \neq j} \frac{1}{\sinh \frac{q_{i j}}{2}} E_{i i} \otimes E_{j i} .
\end{aligned}
$$

It is important to note that $\tilde{R}$ differs from the matrix found in [1] by the term

$$
\frac{1}{2} \sum_{i \neq j} \frac{1}{\sinh \frac{q_{i j}}{2}} E_{i i} \otimes\left(E_{j i}+E_{i j}\right) .
$$

However, this term does not contribute to the bracket (4.64) if we take into account the representation of the $\tilde{\mathcal{L}}$-operator of the trigonometric CM system:

$$
\tilde{\mathcal{L}}=\sum_{i} p_{i} E_{i i}+\frac{1}{2} \sum_{i \neq j} \frac{1}{\sinh \frac{q_{i j}}{2}} E_{i j}
$$


where $(p, q)$ form a pair of canonically conjugated variables. Thus, on the reduced space these matrices define the same Poisson structure for $\tilde{\mathcal{L}}$.

Now we quantize $T^{*} G$ in terms of $A, V$ and $D$ variables. We postulate the following commutation relations

$$
\begin{aligned}
{\left[V_{1}, A_{2}\right] } & =\hbar V_{1} V_{2} s_{12} V_{2}^{-1} \\
{\left[D_{1}, A_{2}\right] } & =-\hbar D_{1} V_{2} \sum_{i} E_{i i} \otimes E_{i i} V_{2}^{-1}
\end{aligned}
$$

One can check that the compatibility of these relations with (3.29) follows from the following identity satisfied by $s_{12}$ :

$$
s_{12}-D_{1}^{-1} s_{12} D_{1}+\sum_{i} E_{i i} \otimes E_{i i}=C
$$

By using eqs.(3.28), (4.67) and (4.68) one derives the commutation relations for the quantum $\mathcal{L}$-operator:

$$
\begin{aligned}
{\left[\mathcal{L}_{1}, \mathcal{L}_{2}\right] } & =\hbar\left[\tilde{r}_{12}, \mathcal{L}_{1}\right]-\hbar\left[\tilde{r}_{21}, \mathcal{L}_{2}\right]+\hbar^{2}\left[\tilde{r}_{12}, \tilde{r}_{21}\right] \\
{\left[D_{1}, \mathcal{L}_{2}\right] } & =-\hbar D_{1} \sum_{i} E_{i i} \otimes E_{i i} \\
{\left[V_{1}, \mathcal{L}_{2}\right] } & =\hbar V_{1} s_{12} .
\end{aligned}
$$

The relation (4.69) can be also written in the form (3.56):

$$
\left[\mathcal{L}_{1}+\hbar \tilde{r}_{21}, \mathcal{L}_{2}+\hbar \tilde{r}_{12}\right]=0
$$

One can check without problems that the $L$-operator (4.66) realizes the representation of the algebra (4.72).

To complete our discussion let us show the existence of $N$ mutually commuting operators in the algebra composed by the $L$-operator and the coordinates $D$. Obviously, $I_{n}=\operatorname{tr} A^{n}$ mutually commute. Applying the technique used in the previous section to derive the quantum integrals of motion, one can show that $I_{n}$ can be expressed as the following function of $\mathcal{L}$ and $D$ :

$$
I_{n}=\operatorname{tr} A^{n}=\operatorname{tr} V \mathcal{L}^{n} V^{-1}=\operatorname{tr}_{12} C_{12}^{t_{2}}\left(\mathcal{L}_{1}+\hbar s_{21}^{t_{2}}\right)^{n} .
$$

In the component form these integrals look as

$$
I_{n}=\left(\mathcal{L}_{j_{1} j_{2}} \delta_{j_{1} m_{1}}+\hbar \frac{\delta_{j_{1} j_{2}}-1}{D_{j_{1}} D_{j_{m_{1}}}^{-1}-1}\right) \ldots\left(\mathcal{L}_{j_{n} j_{n+1}} \delta_{j_{n} m_{n}}+\hbar \frac{\delta_{m_{n-1} j_{n+1}}-\delta_{m_{n-1} j_{n}}}{D_{j_{n}} D_{j_{m_{n}}}^{-1}-1}\right) \delta_{j_{n+1} m_{n}}
$$

Let us note that $I_{n}$ can not be expressed as a linear combination of $\operatorname{tr} \mathcal{L}^{n}$ solely.

\section{Conclusion}

The approach for $R$-matrix quantization of the RS models proposed in the paper seems to be general. The problem of a real interest is to apply it to the trigonometric and elliptic cases. As was recently shown the trigonometric and elliptic RS models are obtained from the cotangent bundles over the centrally extended loop group [19 and double loop group [17] 
respectively. The natural suggestion is to use for this purpose the above-mentioned phase spaces.

It is known that the Heisenberg double [26] can be regarded as a natural deformation of the cotangent bundle $T^{*} G$. It seems to be interesting to investigate the Poisson structure of the Heisenberg double in the same parameterization. One could expect the appearance of another Poisson structure on the Frobenius group induced by the one on the dual Poisson-Lie group $G^{*}$.

The appearance of the quantum Frobenius group $F$ states the problem of developing the corresponding representation theory. Owing to the method of orbits one can suggest that irreducible representations of $F$ should be in correspondence with the symplectic leaves of the Poisson-Lie structure. On the other hand, it is known [27] that the symplectic leaves of a Poisson-Lie structure are the orbits of the dressing transformation. Studying the orbits of $F^{*}$ and corresponding representations of $F$ one can hope to obtain the quantum integrable systems being some "spin" generalizations of the RS model [28]. Another open problem related to the representation theory is to find the universal Frobenius $R$-matrix.

As was shown in [29] the Ruijsenaars Hamiltonians can be related to the special $L$ operator satisfying the fundamental relation $R L L=L L R$ with Belavin's elliptic $R$-matrix. It would be interesting to clarify the relationship of this approach with our construction.

ACKNOWLEDGMENT The authors are grateful to L.O.Chekhov, P.B.Medvedev and N.A.Slavnov for valuable discussions. This work is supported in part by the RFFR grants N96-01-00608 and N96-01-00551 and by the ISF grant a96-1516.

\section{References}

[1] J.Avan and M.Talon, Phys.Lett.B303 (1993) 33-37.

[2] O.Babelon and C.M.Viallet, Phys.Lett.B237 (1989) 411.

[3] J.Avan, O.Babelon and M.Talon, Alg.Anal. 6(2) (1994) 67.

[4] E.K.Sklyanin, Alg.Anal., 6(2) (1994) 227.

[5] H.W.Braden and T.Suzuki, Lett.Math.Phys. 30 (1994) 147.

[6] J.Avan and G.Rollet, The classical r-matrix for the relativistic Ruijsenaars-Schneider system, preprint BROWN-HET-1014 (1995).

[7] Yu.B.Suris, Why are the rational and hyperbolic Ruijsenaars-Schneider hierarchies governed by the same $R$-matrix as the Calogero-Moser ones ? hep-th/9602160.

[8] F.W.Nijhoff, V.B.Kuznetsov, E.K.Sklyanin and O.Ragnisco, Dynamical $r$-matrix for the elliptic Ruijsenaars-Schneider model, solv-int/9603006.

[9] Yu.B.Suris, Elliptic Ruijsenaars-Schneider and Calogero-Moser hierarchies are governed by the same $r$-matrix, solv-int/9603011.

[10] S.N.Ruijsenaars, Comm.Math.Phys. 110 (1987) 191.

[11] J.Avan, O.Babelon and E.Billey, The Gervais-Neveu-Felder equation and the quantum Calogero-Moser systems, preprint PAR LPTHE 95-25, May 1995; hep-th/9505091 (to appear in Comm.Math.Phys.). 
[12] J.L.Gervais and A.Neveu, Nucl.Phys. B238 (1984) 125.

[13] G.Felder, Conformal field theory and integrable systems associated to elliptic curves, hepth/9407154.

[14] O.Babelon, D.Bernard and E.Billey, A quasi-Hopf algebra interpretation of quantum 3-j and 6-j symbols and difference equations, preprint PAR LPTHE 95-51, IHES/P/95/91, q$\operatorname{alg} / 9511019$.

[15] G.E.Arutyunov and P.B.Medvedev, Generating equation for $r$-matrices related to dynamical systems of Calogero type, hep-th/9511070, to appear in Phys.Lett.A.

[16] G.E.Arutyunov, S.A.Frolov and P.B.Medvedev, Elliptic Ruijsenaars-Schneider model via the Poisson reduction of the affine Heisenberg double, hep-th/9607170.

[17] G.E.Arutyunov, S.A.Frolov and P.B.Medvedev Elliptic Ruijsenaars-Schneider model from the cotangent bundle over the two-dimensional current group, hep-th/9608013.

[18] A.Alekseev and L.D.Faddeev, Comm.Math.Phys. 141 (1991) 413.

[19] Gorsky A. and Nekrasov N., Nucl.Phys. B414 (1994) 213; Nucl.Phys. B436 (1995) 582; A.Gorsky, Integrable many body systems in the field theories, Prep. UUITP-16/94, (1994).

[20] L.D.Faddeev Integrable models in (1+1)-dimensional quantum field theory. - In "Recent advances in field theory and statistical mechanics". Eds. Zuber J.B., Stord R. (Les Houches Summer School Proc. session XXXiX, 1982), Elsvier Sci.Publ., (1984) 561.

[21] P.P.Kulish, E.K.Sklyanin, Quantum spectral transform method. Recent developments. - In "Integrable quantum field theories". Eds. Hietarinta J., Montonen C., Lect.Not.Phys. 51 (1982) 61.

[22] M.A.Olshanetsky, A.M.Perelomov, Phys. Reps. 71 (1981) 313.

[23] A.A.Belavin and V.G.Drinfel'd, Funk.Anal.i ego pril. 16(3) (1982) 1-29.

[24] V.Chari and A.Pressley, A Guide to Quantum Groups, Cambridge University Press.

[25] J.M.Maillet, Phys.Lett.B245 (1990) 480.

[26] M.A.Semenov-Tian-Shansky, Teor.Math.Phys. 93 (1992) 302 (in Russian).

[27] M.A.Semenov-Tian-Shansky, Publ.RIMS Kyoto Univ. 21(6) (1985) 1237-1260.

[28] I.Krichever, A.Zabrodin, Spin generalizations of the Ruijsenaars-Schneider model, non-abelian 2D Toda chain and representations of Sklyanin algebra, hep-th/9505039.

[29] K.Hasegawa, Ruijsenaars' commuting difference operators as commuting transfer matrices, q-alg/9512029. 